\title{
Quaternary Message Passing Decoding of LDPC Codes: Density Evolution Analysis and Error Floor
}

\author{
Emna Ben Yacoub ${ }^{\ddagger}$, Balazs Matuz ${ }^{\dagger}$, Alexandre Graell i Amat*, Gianluigi Liva ${ }^{\dagger}$ \\ ${ }^{\ddagger}$ Institute for Communications Engineering, Technical University of Munich, Germany \\ ${ }^{\dagger}$ Institute of Communications and Navigation, German Aerospace Center (DLR), Germany \\ * Department of Electrical Engineering, Chalmers University of Technology, Sweden
}

\begin{abstract}
We revisit a coarsely quantized message passing decoding algorithm for low-density parity-check (LDPC) code ensembles, named quaternary message passing (QMP). Particularly, we analyze the performance of unstructured LDPC codes under QMP decoding by means of density evolution. The impact of degree- 2 and degree- 3 variable nodes on the error floor performance is also discussed. We design a code for QMP that performs within $0.55 \mathrm{~dB}$ of the $5 \mathrm{G}$ LDPC code at a block error rate of $10^{-4}$.
\end{abstract}

\section{INTRODUCTION}

Efficient, low-complexity forward error correction decoders play a crucial role in emerging high-throughput applications. For codes relying on iterative message passing decoding, the internal decoder data flow, defined as the number of bits that are processed in each decoder iteration, scales linearly in the number of quantization bits used to represent the messages exchanged within the decoder [1]. For low-density paritycheck (LDPC) code decoders with messages quantized to four or five bits, data rates in the order of hundreds of Gbps are currently achievable in current hardware implementations, with minor losses in error rate performance with respect to the unquantized case (see, e.g., [2]-[4]).

A coarser quantization of the messages, e.g., with one or two bits, may enable supporting even higher data rates, while posing new challenges to the code/decoder design. The design of coarsely quantized LDPC decoders has attracted considerable attention [5]-[10]. In [8], the authors developed an algorithm with binary messages, referred to as binary message passing (BMP) decoding, which allows to exploit the channel soft information while retaining the one-bit message representation of the Gallager A and Gallager B decoding algorithms [11]. An extension of BMP to ternary and quaternary message alphabets was studied in [9], [10]. The two algorithms were dubbed ternary message passing (TMP) and quaternary message passing (QMP), respectively. Quantizing messages to two bits comes at the cost of a performance loss, which is limited to a few tenths of a dB for high code rates [9], [10], the code rate regime that is of interest for high-speed optical communications [1].

In BMP, TMP, and QMP decoders, the check node $(\mathrm{CN})$ to variable node $(\mathrm{VN})$ messages are modeled as hard observations of an extrinsic discrete memoryless channel (DMC). The VNs can turn these observations into soft messages if the transition probabilities of the extrinsic channel are known. In [8], it was suggested to employ density evolution (DE) to estimate the transition probabilites of the extrinsic DMCs, showing that this is an effective approach, i.e., it provides good estimates down to moderate block lengths. The principle was then extended in [9], [10] to TMP, and QMP decoding.

In this work, we analyze in more detail the performance of QMP decoders for LDPC codes drawn from unstructured irregular code ensembles. In particular, we derive the DE analysis for unstructured irregular LDPC code ensembles. Following the observations of [8], we further investigate the role of degree- 2 and degree- 3 VNs in the error floor performance by performing a trapping sets analysis. This result is particularly insightful, since the design of LDPC codes is often performed for unquantized, unsaturated message passing algorithms. As a consequence, such codes may perform poorly when used in combination with decoders employing coarsely quantized messages. We finally design LDPC codes under QMP and show that a properly designed code performs within $0.55 \mathrm{~dB}$ of the 5G LDPC code (decoded using belief propagation (BP)) at block error rate (BLER) $10^{-4}$.

The rest of the paper is organized as follows. Section II provides some preliminary definitions. QMP decoding is reviewed in Section III. The DE analysis for unstructured LDPC code ensembles under QMP decoding is developed in Section IV. The role of degree-2 and degree- 3 VNs in the error floor region is discussed in Section V, while numerical results are provided in Section VI. Conclusions follow in Section VII.

\section{PRELiminARies}

\section{A. System Model}

We consider binary transmission over the additive white Gaussian noise (AWGN) channel. Denoting the channel input by $X \in\{-1,+1\}$, the channel output is $Y=X+N$, where the noise term $N$ is modeled as zero-mean Gaussian random variable with variance $\sigma^{2}$. We define the signal-to-noise ratio as $E_{\mathrm{b}} / N_{0}$, where $E_{\mathrm{b}}$ is the energy per information bit and $N_{0}$ the single-sided noise power spectral density. We have $E_{\mathrm{b}} / N_{0}=\left(2 R \sigma^{2}\right)^{-1}$, with $R$ being the rate of the code.

\section{B. Low-Density Parity-Check Codes}

We denote by $G=(\mathcal{V} \cup \mathcal{C}, \mathcal{E})$ the Tanner graph of an LDPC code, consisting of a set $\mathcal{V}$ of $n \mathrm{VNs}$, a set $\mathcal{C}$ of $m \mathrm{CNs}$, and a set of edges $\mathcal{E}=\left\{e_{i j}\right\}$, where $e_{i j}$ is an edge between $\mathrm{VN} \mathrm{v}_{j} \in \mathcal{V}$ and $\mathrm{CN} \mathrm{c}_{i} \in \mathcal{C}$. The set of neighbors of $\mathrm{VN}$ 


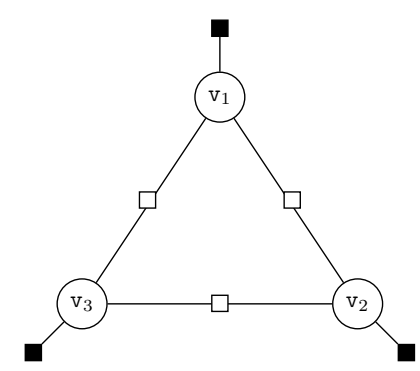

Fig. 1. Subgraph induced by elementary trapping set $\mathcal{T}_{33}^{\mathrm{e}}$ of a regular $(3,6)$ code with girth 6 . The three VNs, the three even degree CNs and the respective edges connecting them form a cycle.

$\mathrm{v}_{j}$ and $\mathrm{CN} \mathrm{c}_{i}$ are denoted by $\mathcal{N}\left(\mathrm{v}_{j}\right)$ and $\mathcal{N}\left(\mathrm{c}_{i}\right)$, respectively. The edge-oriented degree distribution polynomials of an LDPC code graph are

$$
\lambda(x)=\sum_{i} \lambda_{i} x^{i-1} \quad \text { and } \quad \rho(x)=\sum_{i} \rho_{i} x^{i-1}
$$

where $\lambda_{i}$ and $\rho_{i}$ correspond to the fraction of edges incident to $\mathrm{VNs}$ and $\mathrm{CNs}$ with degree $i$, respectively.

An unstructured irregular LDPC code ensemble $\mathscr{C}_{n}^{\lambda, \rho}$ is the set of length- $n$ linear block codes defined by all possible Tanner graphs with $n$ VNs and degree distributions $\lambda(x)$ and $\rho(x)$.

\section{Trapping Sets}

For a set $\mathcal{S} \subseteq \mathcal{V}$, let $\mathcal{N}(\mathcal{S})$ be the set of its neighboring CNs. Furthermore, we denote by $\mathcal{N}_{\mathrm{o}}(\mathcal{S})$ the set of CNs in $\mathcal{N}(\mathcal{S})$ that are connected to $\mathcal{S}$ an odd number of times and by $\mathcal{N}_{\mathrm{e}}(\mathcal{S})$ the set of CNs in $\mathcal{N}(\mathcal{S})$ that are connected to $\mathcal{S}$ an even number of times.

Definition 1 (Trapping set). An $(a, b)$ trapping set $\mathcal{T}_{a, b}$ is a set of $a$ VNs for which $\mathcal{N}_{\mathrm{o}}\left(\mathcal{T}_{a, b}\right)$ contains $b$ CNs [12].

Definition 2 (Elementary trapping set). An elementary trapping set $\mathcal{T}_{a, b}^{\mathrm{e}}$ is a trapping set where each $\mathrm{CN} \mathrm{c} \in \mathcal{N}_{\mathrm{e}}\left(\mathcal{T}_{a, b}^{\mathrm{e}}\right)$ is connected to two VNs in $\mathcal{T}_{a, b}^{\mathrm{e}}$ and each $\mathrm{CN} \mathrm{c} \in \mathcal{N}_{\mathrm{o}}\left(\mathcal{T}_{a, b}^{\mathrm{e}}\right)$ is connected to exactly one $\mathrm{VN}$ in $\mathcal{T}_{a, b}^{\mathrm{e}}$.

Definition 3 (Induced subgraph). A subgraph $G^{\prime}$ of $G$ induced by the set of VNs $\mathcal{S}$ is a bipartite graph composed of the VNs in $\mathcal{S}$, their neighboring CNs, and the edges connecting these CNs to the VNs in $\mathcal{S}$.

Example 1. A subgraph induced by an elementary trapping set $\mathcal{T}_{3,3}^{\mathrm{e}}$ is shown in Fig. 1. The trapping set consists of $a=3$ VNs. The $b=3 \mathrm{CNs}$ in $\mathcal{N}_{\mathrm{o}}\left(\mathcal{T}_{3,3}^{\mathrm{e}}\right)$ are represented by filled black squares while the three CNs in $\mathcal{N}_{\mathrm{e}}\left(\mathcal{T}_{3,3}^{\mathrm{e}}\right)$ are depicted with white squares.

\section{Quaternary Message Passing Decoding}

We review next the QMP decoding algorithm introduced in [10]. Let us denote by $m_{\mathrm{c} \rightarrow \mathrm{v}}^{(\ell)}$ the message sent from CN c to VN v during the $\ell$-th iteration. Similarly, $m_{\mathrm{v} \rightarrow c}^{(\ell)}$ is the message sent from VN v to $\mathrm{CN} c$ at the $\ell$-th iteration. The alphabet of the messages exchanged between $\mathrm{CNs}$ and $\mathrm{VNs}$ is $\mathcal{M} \triangleq\{-\mathrm{H},-\mathrm{L},+\mathrm{L},+\mathrm{H}\}$, where the symbols $\pm \mathrm{L}$ and $\pm \mathrm{H}$ represent messages with low and high reliability, respectively. We will refer next to $\mathrm{L}$ and $\mathrm{H}$ as message magnitudes or message reliabilities. Moreover, with some abuse of notation, we will denote the magnitude of a generic message $m \in \mathcal{M}$ as $|m|$.

At the first iteration, each $\mathrm{VN}$ sends to its neighboring $\mathrm{CNs}$ the message

$$
m_{\mathrm{v} \rightarrow \mathrm{c}}^{(0)}=\Psi\left(L_{\mathrm{ch}}\right)
$$

where

$$
L_{\mathrm{ch}}=\frac{2}{\sigma^{2}} y
$$

is the channel log-likelihood ratio (LLR) and $\Psi(\cdot)$ denotes the message quantization function

$$
\Psi(x)= \begin{cases}-\mathrm{H} & x \leq-T \\ -\mathrm{L} & -T<x<0 \\ +\mathrm{L} & 0 \leq x<T \\ +\mathrm{H} & x \geq T .\end{cases}
$$

In (2), $T$ is a non-negative quantization threshold, whose value is kept constant across iterations. We use DE (see Section IV) to select the value of $T$ that minimizes the iterative decoding threshold of the respective LDPC code ensemble. The CN update is

$$
m_{\mathrm{c} \rightarrow \mathrm{v}}^{(\ell)}=\min _{\mathrm{v}^{\prime} \in \mathcal{N}(\mathrm{c}) \backslash \mathrm{v}}\left|m_{\mathrm{v}^{\prime} \rightarrow \mathrm{c}}^{(\ell-1)}\right| \prod_{\mathrm{v}^{\prime} \in \mathcal{N}(\mathrm{c}) \backslash \mathrm{v}} \operatorname{sign}\left(m_{\mathrm{v}^{\prime} \rightarrow \mathrm{c}}^{(\ell-1)}\right) .
$$

At the $\ell$-th iteration, the outgoing VN message is

$$
m_{\mathrm{v} \rightarrow \mathrm{c}}^{(\ell)}=\Psi\left(L_{\mathrm{ch}}+\sum_{\mathrm{c}^{\prime} \in \mathcal{N}(\mathrm{v}) \backslash \mathrm{c}} L_{\mathrm{ex}}\left(m_{\mathrm{c}^{\prime} \rightarrow \mathrm{v}}^{(\ell)}\right)\right)
$$

and QMP proceeds by iterating (4)-(3) until the decoder succeeds in converging to a codeword, or a maximum number of iterations $\ell_{\max }$ is reached. The final hard decision at each $\mathrm{VN}$ is

$$
\hat{x}=\operatorname{sign}\left(L_{\mathrm{ch}}+\sum_{\mathrm{c}^{\prime} \in \mathcal{N}(\mathrm{v})} L_{\mathrm{ex}}\left(m_{\mathrm{c}^{\prime} \rightarrow \mathrm{v}}^{(\ell)}\right)\right) .
$$

In (4) and (5), $L_{\mathrm{ex}}(\cdot)$ is the LLR associated to the extrinsic channel used to model the CN-to-VN message, and it is defined as

$$
L_{\mathrm{ex}}\left(m_{\mathrm{c}^{\prime} \rightarrow \mathrm{v}}^{(\ell)}\right)=w^{(\ell)}\left(\left|m_{\mathrm{c}^{\prime} \rightarrow \mathrm{v}}^{(\ell)}\right|\right) \operatorname{sign}\left(m_{\mathrm{c}^{\prime} \rightarrow \mathrm{v}}^{(\ell)}\right),
$$

where, in general, the real-valued reliability term $w^{(\ell)}(\cdot)$ depends on the iteration number and on the communication channel nuisance. Throughout this work, we will use DE [10] to estimate $w^{(\ell)}(\cdot)$ (see (6) in Section IV). Due to the unappealing complexity cost entailed by adapting the reliability terms $w^{(\ell)}(\cdot)$ to the underlying communication channel signal-to-noise ratio (SNR), we will keep the reliability terms 
constant over the whole SNR range for the simulation results in Sections V and VI. The reliability terms that are used are those obtained via DE at the iterative decoding threshold of the respective code ensemble.

\section{DEnsity EVOLUTION ANALysis}

In this section, we outline DE analysis of the QMP decoder for unstructured LDPC code ensembles. Due to the channel symmetry and to the code linearity, we can assume the transmission of the all-zero codeword. Under binary antipodal mapping, this is equivalent to assuming the channel input $X=+1$. Let $M_{\mathrm{v} \rightarrow \mathrm{c}}^{(\ell)}$ be the random variable (RV) associated to VN-to-CN messages during the $\ell$-th iteration. Similarly, $M_{\mathrm{c} \rightarrow \mathrm{v}}^{(\ell)}$ represents the RV associated to $\mathrm{CN}$-to- $\mathrm{VN}$ messages. We denote by $p_{m}^{(\ell)}$ the probability that $M_{\mathrm{v} \rightarrow \mathrm{c}}^{(\ell)}$ takes the value $m \in \mathcal{M}$. Similarly, we denote by $q_{m}^{(\ell)}$ the probability that $M_{\mathrm{c} \rightarrow \mathrm{v}}^{(\ell)}$ takes the value $m \in \mathcal{M}$. Since $p_{+\mathrm{H}}^{(\ell)}=1-p_{-\mathrm{H}}^{(\ell)}-p_{-\mathrm{L}}^{(\ell)}-p_{+\mathrm{L}}^{(\ell)}$ and $q_{+\mathrm{H}}^{(\ell)}=1-q_{-\mathrm{H}}^{(\ell)}-q_{-\mathrm{L}}^{(\ell)}-q_{+\mathrm{L}}^{(\ell)}$, we only need to track the probabilities $p_{m}^{(\ell)}$ and $q_{m}^{(\ell)}$ for $m \in\{-\mathrm{H},-\mathrm{L}, \mathrm{L}\}$. In the limit of infinite block length, $n \rightarrow \infty$, the evolution of the message distributions can be tracked as follows.

1) Initialization. Conditioned on $X=+1$, the channel LLRs are Gaussian RVs with mean $\mu_{\mathrm{ch}}=4 R E_{\mathrm{b}} / N_{0}$ and variance $\sigma_{\mathrm{ch}}^{2}=2 \mu_{\mathrm{ch}}$. Therefore, recalling (1), we have

$$
\begin{aligned}
& p_{-\mathrm{H}}^{(0)}=\mathbb{P}\left(L_{\mathrm{ch}} \leq-T\right)=Q\left(\frac{T+\mu_{\mathrm{ch}}}{\sigma_{\mathrm{ch}}}\right) \\
& p_{-\mathrm{L}}^{(0)}=Q\left(\frac{\mu_{\mathrm{ch}}}{\sigma_{\mathrm{ch}}}\right)-Q\left(\frac{T+\mu_{\mathrm{ch}}}{\sigma_{\mathrm{ch}}}\right) \\
& p_{+\mathrm{L}}^{(0)}=Q\left(\frac{-T+\mu_{\mathrm{ch}}}{\sigma_{\mathrm{ch}}}\right)-Q\left(\frac{\mu_{\mathrm{ch}}}{\sigma_{\mathrm{ch}}}\right)
\end{aligned}
$$

where the $Q$-function is the tail distribution of a standard normal RV.

2) For $\ell=1,2, \ldots, \ell_{\max }$

Check-to-variable node update.

$$
\begin{aligned}
q_{-\mathrm{H}}^{(\ell)}= & \frac{1}{2}\left[\rho\left(1-p_{-\mathrm{L}}^{(\ell-1)}-p_{+\mathrm{L}}^{(\ell-1)}\right)\right. \\
& \left.-\rho\left(1-2 p_{-\mathrm{H}}^{(\ell-1)}-p_{-\mathrm{L}}^{(\ell-1)}-p_{+\mathrm{L}}^{(\ell-1)}\right)\right] \\
q_{-\mathrm{L}}^{(\ell)}= & \frac{1}{2}\left[1+\rho\left(1-2 p_{-\mathrm{H}}^{(\ell-1)}-p_{-\mathrm{L}}^{(\ell-1)}-p_{+\mathrm{L}}^{(\ell-1)}\right)\right. \\
& -\rho\left(1-p_{-\mathrm{L}}^{(\ell-1)}-p_{+\mathrm{L}}^{(\ell-1)}\right)- \\
& \left.\rho\left(1-2 p_{-\mathrm{H}}^{(\ell-1)}-2 p_{-\mathrm{L}}^{(\ell-1)}\right)\right] \\
q_{+\mathrm{L}}^{(\ell)}= & \frac{1}{2}\left[1-\rho\left(1-2 p_{-\mathrm{H}}^{(\ell-1)}-p_{-\mathrm{L}}^{(\ell-1)}-p_{+\mathrm{L}}^{(\ell-1)}\right)\right. \\
& -\rho\left(1-p_{-\mathrm{L}}^{(\ell-1)}-p_{+\mathrm{L}}^{(\ell-1)}\right) \\
& \left.+\rho\left(1-2 p_{-\mathrm{H}}^{(\ell-1)}-2 p_{-\mathrm{L}}^{(\ell-1)}\right)\right] .
\end{aligned}
$$

Variable-to-check node update.

$$
\begin{aligned}
p_{-\mathrm{H}}^{(\ell)}= & \sum_{d} \lambda_{d} \mathbb{P}\left(L_{\mathrm{in}}^{(\ell)}=l_{\mathrm{in}}\right) Q\left(\frac{T+l_{\mathrm{in}}+\mu_{\mathrm{ch}}}{\sigma_{\mathrm{ch}}}\right) \\
p_{-\mathrm{L}}^{(\ell)}= & \sum_{d} \lambda_{d} \mathbb{P}\left(L_{\mathrm{in}}^{(\ell)}=l_{\mathrm{in}}\right) \times \\
& {\left[Q\left(\frac{l_{\mathrm{in}}+\mu_{\mathrm{ch}}}{\sigma_{\mathrm{ch}}}\right)-Q\left(\frac{T+l_{\mathrm{in}}+\mu_{\mathrm{ch}}}{\sigma_{\mathrm{ch}}}\right)\right] } \\
p_{+\mathrm{L}}^{(\ell)}= & \sum_{d} \lambda_{d} \mathbb{P}\left(L_{\mathrm{in}}^{(\ell)}=l_{\mathrm{in}}\right) \times \\
& {\left[Q\left(\frac{-T+l_{\mathrm{in}}+\mu_{\mathrm{ch}}}{\sigma_{\mathrm{ch}}}\right)-Q\left(\frac{l_{\mathrm{in}}+\mu_{\mathrm{ch}}}{\sigma_{\mathrm{ch}}}\right)\right] }
\end{aligned}
$$

where

$$
w^{(\ell)}(\mathrm{L})=\ln \left(\frac{q_{+\mathrm{L}}^{(\ell)}}{q_{-\mathrm{L}}^{(\ell)}}\right), \quad w^{(\ell)}(\mathrm{H})=\ln \left(\frac{q_{+\mathrm{H}}^{(\ell)}}{q_{-\mathrm{H}}^{(\ell)}}\right),
$$

and $L_{\text {in }}^{(\ell)}$ is a RV modeling the sum of the LLRs for the $d-1$ messages received by a $\mathrm{VN}$ and sent from $d-1$ neighboring $\mathrm{CNs}$ at the $\ell$-th iteration. We have

$$
\begin{aligned}
\mathbb{P}\left(L_{\text {in }}^{(\ell)}=l_{\text {in }}\right)= & \sum_{u, v, e}\left(\begin{array}{c}
d-1 \\
u, v, e, d-1-u-v-e
\end{array}\right) \times \\
& \left(q_{-\mathrm{H}}^{(\ell)}\right)^{u}\left(q_{-\mathrm{L}}^{(\ell)}\right)^{v}\left(q_{+\mathrm{L}}^{(\ell)}\right)^{e} \times \\
& \left(1-q_{-\mathrm{H}}^{(\ell)}-q_{-\mathrm{L}}^{(\ell)}-q_{+\mathrm{L}}^{(\ell)}\right)^{d-1-u-v-e}
\end{aligned}
$$

where the sum is over integer values $u, v, e$ satisfying $0 \leq u+v+e \leq d-1$, and $l_{\text {in }}=(e-v) w^{(\ell)}(\mathrm{L})+(d-$ $1-e-v-2 u) w^{(\ell)}(\mathrm{H})$.

The ensemble iterative decoding threshold $\left(E_{\mathrm{b}} / N_{0}\right)^{\star}$ is defined as the minimum $E_{\mathrm{b}} / N_{0}$ for which $p_{-\mathrm{H}}^{(\ell)} \rightarrow 0$ and $p_{-\mathrm{L}}^{(\ell)} \rightarrow 0$ as $\ell \rightarrow \infty$.

\section{ERROR FLOOR ANALYSIS FOR QMP DECODING}

The error floor of an LDPC code under message-passing decoding is often due to short cycles in the code's Tanner graph. We are interested in analyzing the evolution of the messages within short cycles. We introduce the concept of cycle-extrinsic messages (CEMs) and cycle-intrinsic messages (CIMs) below.

Definition 4 (CEM). A CEM is a message received by a VN (CN) in a cycle such that the message is originated by a $\mathrm{CN}$ (VN) which is not part of the cycle.

Definition 5 (CIM). A CIM is a message received by a VN $(\mathrm{CN})$ in a cycle such that the message is originated by a $\mathrm{CN}$ (VN) which is part of the cycle.

Consider a cycle with $a \mathrm{VNs}$ and $b$ CNs comprising only degree-2 and degree-3 VNs. We have that the set of $a$ VNs forms an elementary trapping set $\mathcal{T}_{a, b}^{\mathrm{e}}$. Cycles in a code's Tanner graph comprising only degree-2 and/or degree-3 VNs are particularly harmful for BMP decoding [8]; whenever all VNs in the cycle receive erroneous channel LLRs, BMP 


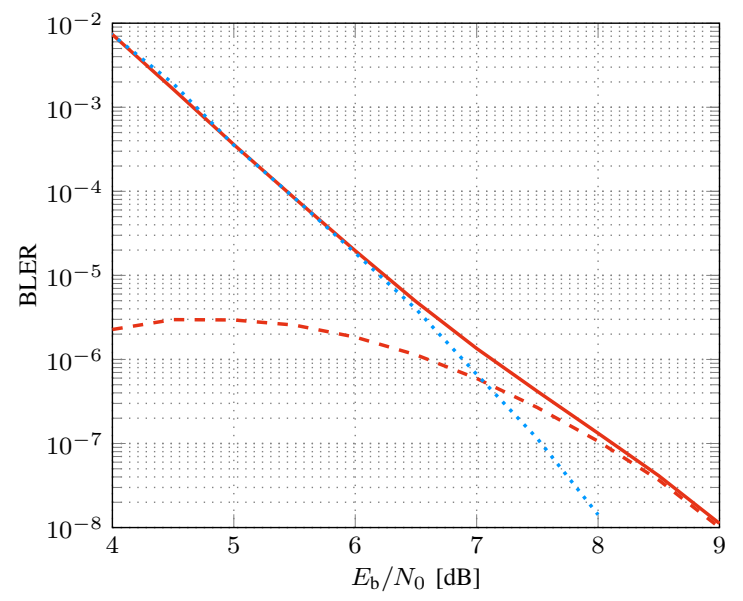

Fig. 2. BLER versus $E_{\mathrm{b}} / N_{0}$ for code $\mathcal{C}_{1}$ : performance under QMP decoding with 25 iterations is given by the red solid line ( - ), the contribution to the error probability of type- $\xi$ error events is given by the red dashed line (- - - ), the performance with 20 iterations, followed by 5 additional iterations with modified channel LLRs is given by the blue dotted line ( $\ldots \ldots)$.

decoding may fail. This is the case, for instance, if also all CNs in the cycle receive correct CEMs for all iterations [8, Thm. 3]. Next, we illustrate that cycles comprising degree-2 and/or degree-3 VNs are also harmful for QMP decoding. However, the conditions on a decoding failure are more involved than for BMP decoding as illustrated by the following lemma.

Lemma 1. Consider a cycle involving only degree-2 and/or degree-3 VNs so that all VNs in the cycle receive erroneous channel LLRs $L_{\mathrm{ch}}$ with $\left|\Psi\left(L_{\mathrm{ch}}\right)\right|=\mathrm{H}$. If from iteration $\ell$ onward all CEMs are correct with reliability $\mathrm{H}$ and at the beginning of iteration $\ell$ the CIMs received by the VNs in the cycle are erroneous with reliability H, a QMP decoder will not be able to correct the erroneous VNs in the cycle.

Proof. At the beginning of iteration $\ell$ each $\mathrm{VN}$ in the cycle will receive an erroneous channel LLR, two erroneous CIMs with reliability $\mathrm{H}$, and, in the case of a degree three $\mathrm{VN}$, one correct CEM with reliability $\mathrm{H}$ (see Fig. 1). By (4), the outgoing $\mathrm{VN}$ messages will be all erroneous with reliability $\mathrm{H}$, and consequently the hard decision in (5) will also be erroneous. Further, under the assumption of correct highly reliable CEMs, all CNs in the cycle will simply forward the outgoing messages from the VNs in the cycle. Hence, the CIMs at the VNs at iteration $\ell+1$ remain erroneous with reliability $\mathrm{H}$, as at iteration $\ell$.

Informally, if the VNs forming a cycle (where all VNs have degree two and/or three) receive high-magnitude erroneous channel LLRs, the QMP decoder may fail in correcting the errors. An empirical evidence on the impact of these events on the error floor performance of LDPC codes with large fractions of degree-3 VNs is provided in the following example.

Example 2. Fig. 2 illustrates the BLER performance under QMP decoding of a short $(3,6)$ LDPC code with parameters $(120,60)$ and girth 6 , denoted by $\mathcal{C}_{1}$. Additionally, decoding failures due to a specific type of error events, referred to as

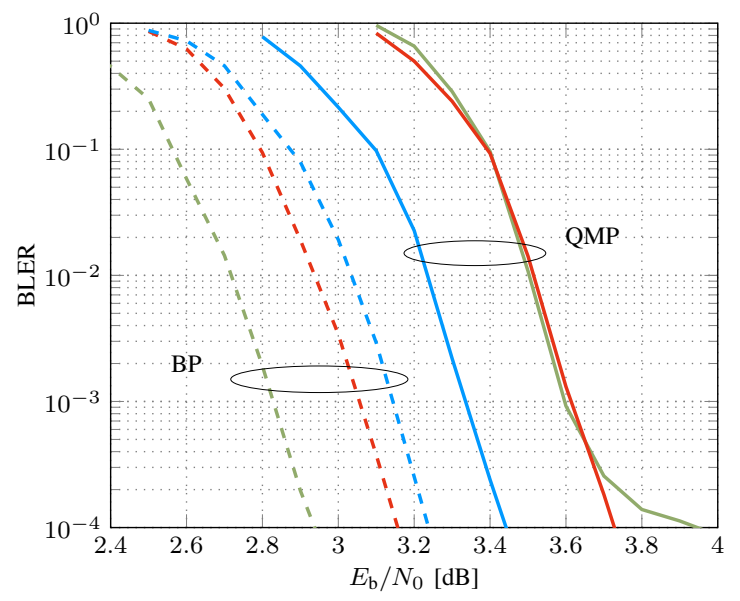

Fig. 3. BLER versus $E_{\mathrm{b}} / N_{0}$ for code $\mathcal{C}_{2}$ (-/---), AR4JA code ( - /- - - ) and 5G LDPC code (- /- - - ) under QMP decoding (solid lines) and BP decoding (dashed lines).

type- $\xi$, are counted. These events are associated with channel realizations such that the decoded codeword has exactly 3 erroneous bit decisions for which the corresponding VNs are all in $\mathcal{T}_{3,3}^{\mathrm{e}}$, i.e., for which the corresponding VNs are involved in a length- 6 cycle. Further, these events require that for all the erroneous channel, LLRs $\left|\Psi\left(L_{\mathrm{ch}}\right)\right|=\mathrm{H}$, and that all CEM $s$ are correct with reliability $\mathrm{H}$ for the last iteration, while the CIMs at the VNs in $\mathcal{T}_{3,3}^{\mathrm{e}}$ are erroneous with reliability H. Fig. 2 shows the contribution of type- $\xi$ error events to the BLER, which becomes dominant at high $E_{b} / N_{0}$.

From the observations above, we conclude that degree- 2 and degree-3 VNs should be used sparingly in order to avoid high error floors under QMP, i.e., one should introduce suitable constraints on the VN degree distribution.

One can also modify the QMP decoder to mitigate the effects of trapping sets such as those described in Lemma 1. A simple strategy is as follows. After a certain number of iterations one may identify all unsatisfied $\mathrm{CNs}$ and their neighboring VNs, which form a set $\mathcal{S}$. Then, one sets the channel LLRs of the VNs in $\mathcal{S}$ to zero. This is exemplified in Fig. 2, where the channel LLRs for VNs in $\mathcal{S}$ are set to zero after 20 decoding iterations and additional 5 decoding iterations are performed with the new (i.e., zero) channel LLRs. For the code $\mathcal{C}_{1}$ in Example 2, this simple strategy yields a gain of almost $1 \mathrm{~dB}$ at a BLER for $10^{-8}$.

\section{NUMERICAL RESUlTS}

Next, we address the performance of QMP decoding for high-rate LDPC codes whose degree distributions have been designed by means of the DE analysis in Section IV. The maximum VN degree is limited to 20 . In order to avoid cycles involving only degree- 2 and degree- $3 \mathrm{VNs}$, we impose the constraint [8]

$$
3 \lambda_{2}+4 \lambda_{3}<6 / \bar{d}_{c}
$$

where $\bar{d}_{c}$ is the average $\mathrm{CN}$ degree. Table I shows optimized degree distributions for unstructured LDPC code 
TABLE I

DEGREE DISTRIBUTIONS OPTIMIZED FOR QMP DECODING, UNDER THE CONSTRAINT (7). THE OPTIMIZED PARAMETER $T$ IS PROVIDED, ALONG WITH THE ITERATIVE DECODING THRESHOLD AND THE RESPECTIVE SHANNON LIMIT OF THE BINARY-INPUT AWGN CHANNEL.

\begin{tabular}{cccccc}
\hline$R$ & $\lambda(x)$ & $\rho(x)$ & $T$ & $\left(E_{\mathrm{b}} / N_{0}\right)^{\star}[\mathrm{dB}]$ & $\left(E_{\mathrm{b}} / N_{0}\right)_{\mathrm{SH}}[\mathrm{dB}]$ \\
\hline $1 / 2$ & $0.0964 x+0.0899 x^{2}+0.4906 x^{3}+0.3231 x^{19}$ & $0.7637 x^{8}+0.2363 x^{9}$ & 1.7 & 1.48 & 0.19 \\
$2 / 3$ & $0.0216 x+0.0568 x^{2}+0.5615 x^{3}+0.0027 x^{9}+0.3574 x^{19}$ & $0.060 x^{14}+0.9401 x^{15}$ & 1.4 & 2.07 & 1.06 \\
$3 / 4$ & $0.0695 x^{2}+0.5823 x^{3}+0.0029 x^{4}+0.0016 x^{9}+0.3437 x^{19}$ & $0.5605 x^{20}+0.4395 x^{21}$ & 1.5 & 2.47 & 1.63 \\
$4 / 5$ & $0.0487 x^{2}+0.6173 x^{3}+0.0022 x^{9}+0.0056 x^{14}+0.3261 x^{19}$ & $0.3260 x^{25}+0.6740 x^{26}$ & 1.6 & 2.78 & 2.04 \\
$7 / 8$ & $0.0334 x^{2}+0.6143 x^{3}+0.0038 x^{5}+0.0018 x^{9}+0.3468 x^{19}$ & $0.2735 x^{42}+0.7265 x^{43}$ & 1.6 & 3.43 & 2.84 \\
$9 / 10$ & $0.0240 x^{2}+0.6144 x^{3}+0.0128 x^{4}+0.3488 x^{19}$ & $0.9362 x^{54}+0.0638 x^{55}$ & 1.6 & 3.73 & 3.20 \\
\hline
\end{tabular}

ensembles under QMP decoding obtained via differential evolution. The code ensembles have design rates $R \in$ $\{1 / 2,2 / 3,3 / 4,4 / 5,7 / 8,9 / 10\}$. In the table, the respective iterative decoding thresholds $\left(E_{\mathrm{b}} / N_{0}\right)^{\star}$ are also provided together with the Shannon limit of the binary-input AWGN channel, denoted as $\left(E_{\mathrm{b}} / N_{0}\right)_{\mathrm{SH}}$. Observe that for increasing code rate, the gap between the thresholds and the Shannon limit decreases-while for $R=1 / 2$ the gap is around $1.3 \mathrm{~dB}$, it is only $0.6 \mathrm{~dB}$ for $R=7 / 8$. This suggests that QMP decoders are particularly well suited for high-rate LDPC codes, as already observed in [10].

Fig. 3 shows the BLER versus $E_{\mathrm{b}} / N_{0}$ of a $(5120,4096)$ LDPC code of rate $R=4 / 5$, denoted by $\mathcal{C}_{2}$, on the binary-input AWGN channel under BP and QMP decoding with 30 decoding iterations. The degree distribution is taken from Table I. As a comparison, the BLER of the $(5120,4096)$ accumulate-repeat-4-jagged-accumulate (AR4JA) code from [13] is also provided. From the figure one can observe that although the AR4JA code slightly outperforms $\mathcal{C}_{2}$ under BP decoding, it shows a loss of $0.3 \mathrm{~dB}$ under QMP decoding. As a further benchmark, the performance of a $(5376,4224)$ protograph-based 5G-NR LDPC code [14] is also depicted. Although the code has excellent performance under BP decoding, QMP entails a loss of around $0.8 \mathrm{~dB}$ and introduces an error floor above $10^{-4}$. Remarkably, the designed code under QMP decoding performs within 0.55 $\mathrm{dB}$ of the 5G code decoded using BP. Thus, when QMP decoding is considered, dedicated code designs help to obtain both good waterfall end error floor performance with limited losses compared to codes designed for BP decoding. Further, codes designed for BP decoding may show visible losses both in waterfall and error floor performance under QMP decoding. This underlines the need for specific code designs when coarsely quantized decoders are employed.

\section{CONCLUSIONS}

We reviewed a message passing decoding algorithm for lowdensity parity-check codes, employing two-bit quantization for the messages exchanged within the decoder. The decoding algorithm, named quaternary message passing, is analyzed through DE for unstructured LDPC code ensembles. The role of degree- 2 and degree-3 variable nodes in the error floor performance has been discussed. Finite-length simulations show that in the high code rate regime, by properly choosing the degree distributions that specify the code ensemble, it is possible to limit the performance loss due to quantization with respect to unquantized belief propagation decoding to a few tenths of a dB. An intersting future research direction is the comparison of QMP decoding with other coarsely quantized decoding algorithms.

\section{REFERENCES}

[1] B. P. Smith, A. Farhood, A. Hunt, F. R. Kschischang, and J. Lodge, "Staircase Codes: FEC for $100 \mathrm{~Gb} / \mathrm{s}$ OTN," J. Lightw. Technol., vol. 30, no. 1, pp. 110-117, Jan. 2012.

[2] L. Schmalen, V. Aref, J. Cho, D. Suikat, D. Rösener, and A. Leven, "Spatially Coupled Soft-Decision Error Correction for Future Lightwave Systems," J. Lightw. Technol., vol. 33, no. 5, pp. 1109-1116, Mar. 2015.

[3] F. Steiner and G. Kramer, "Optimization of Bit Mapping and Quantized Decoding for Off-the-Shelf Protograph LDPC Codes with Application to IEEE 802.3ca," in Proc. ISTC, Hong Kong, Dec. 2018.

[4] J. Lewandowsky and G. Bauch, "Information-Optimum LDPC Decoders Based on the Information Bottleneck Method," IEEE Access, vol. 6, pp. 4054-4071, Jan. 2018.

[5] S. K. Planjery, D. Declercq, L. Danjean, and B. Vasic, "Finite Alphabet Iterative Decoders-Part I: Decoding Beyond Belief Propagation on the Binary Symmetric Channel," IEEE Trans. Commun., vol. 61, no. 10, pp. 4033-4045, Oct. 2013.

[6] D. Declercq, B. Vasic, S. K. Planjery, and E. Li, "Finite Alphabet Iterative Decoders-Part II: Towards Guaranteed Error Correction of LDPC Codes via Iterative Decoder Diversity," IEEE Trans. Commun., vol. 61, no. 10, pp. 4046-4057, Oct. 2013.

[7] F. Cai, X. Zhang, D. Declercq, S. K. Planjery, and B. Vasic, "Finite Alphabet Iterative Decoders for LDPC Codes: Optimization, Architecture and Analysis," IEEE Trans. Circ. and Syst., vol. 61, no. 5, pp. 1366-1375, May 2014.

[8] G. Lechner, T. Pedersen, and G. Kramer, "Analysis and Design of Binary Message Passing Decoders," IEEE Trans. Commun., vol. 60, no. 3, pp. 601-607, Mar. 2012.

[9] E. Ben Yacoub, F. Steiner, B. Matuz, and G. Liva, "Protograph-based LDPC code design for ternary message passing decoding," in Int. Conf. Syst. Commun. Coding, SCC 2019, Rostock, Germany, Feb. 2019.

[10] F. Steiner, E. Ben Yacoub, B. Matuz, G. Liva, and A. Graell i Amat, "One and two bit message passing for SC-LDPC codes with higherorder modulation," J. Lightw. Techn., vol. 37, no. 23, pp. 5914-5925, Dec 2019.

[11] R. G. Gallager, Low-Density Parity-Check Codes. Cambridge, MA: M.I.T. Press, 1963

[12] O. Milenkovic, E. Soljanin, and P. Whiting, "Asymptotic spectra of trapping sets in regular and irregular LDPC code ensembles," IEEE Trans. Inf. Theory, vol. 53, no. 1, pp. 39-55, Jan 2007.

[13] TM Synchronization and Channel Coding, Blue Book, Issue 2, Consultative Committee for Space Data Systems (CCSDS) Recommendation for Space Data System Standard 131.0.B.2, Aug. 2011.

[14] Technical Specification Group Radio Access Network - NR - Multiplexing and channel coding, 3GPP Technical specification TS 38.212 V16.5.0, Mar. 2021. 